\title{
DE DERECHOS A HECHOS. EL ACCESO DIFERENCIADO ENTRE NACIONALES Y MIGRANTES NICARAGÜENSES A LOS SERVICIOS DE SALUD EN COSTA RICA
}

\section{FROM RIGHTS TO REALITY DIFFERENTIATED ACCESS BETWEEN NATIONALS AND NICARAGUAN MIGRANTS TO COSTA RICA'S HEALTH SERVICES}

Koen VoOrend* \& Rebeca Sura-FonseCA**

Resumen: Como en otras partes del mundo, en Costa Rica existe una tensión sobre la incorporación de migrantes en los servicios de salud públicos, que alimenta la sospecha de que existe un acceso diferenciado a los servicios de salud entre nacionales y migrantes. En este artículo, con base en datos primarios de una encuesta representativa para la población migrante nicaragüense, se analiza el acceso real a los servicios de salud pública de migrantes, y la importancia de la nacionalidad, el estado migratorio y el tener un seguro médico para dicho acceso. Los hallazgos sugieren que el acceso a los servicios de salud pública entre nacionales y migrantes es estratificada, lo cual subraya de manera más general la importancia de analizar la praxis, y no solo los derechos sociales formales.

Palabras clave: Politica social; migración; servicios de salud; déficit de implementación; exclusión; Costa Rica.

* PhD, Profesor Asociado de la Universidad de Costa Rica: investigador del Instituto de Investigaciones Sociales, docente de la Escuela de Ciencias de la Comunicación Colectiva y del posgrado de Comunicación y Desarrollo.

** M.Sc., Profesora de Sociología/Estadística de la Universidad de Costa Rica. Estudiante doctoral del IDAES-UNSAM, Buenos Aires, Argentina. 


\begin{abstract}
Like in other parts of the world, the incorporation of migrants in public health services creates tension in Costa Rica. In this article, based on primary data from a representative survey among Nicaraguan immigrants, we evaluate migrants' actual access to public health services, with special interest in the importance of nationality, immigration status and medical insurance. The findings suggest that access to public health services between nationals and migrants is stratified, which underscores the general importance of analyzing the praxis of social rights, and not just formal entitlements.
\end{abstract}

Key words: Social policy; migration; health services; implementation deficit; exclusion; Costa Rica.

\title{
INTRODUCCIÓN
}

Cuando se trata de derechos sociales y el acceso real a servicios sociales para migrantes, entre el dicho y el hecho puede haber mucho trecho. En Costa Rica, como en otros países, existe una tensión entre el reconocimiento formal de los derechos sociales de migrantes, a través de la incorporación de derechos humanos en las leyes nacionales, y el reconocimiento real de estos derechos en su materialización de la provisión de servicios sociales para dicha población. Esta tensión se nutre de voces de chovinismo de bienestar, que abogan por la creación de barreras al acceso a servicios sociales con el fin de reservarlos para la población nacional (Banting, 2000; Morrisens, 2008).

En un contexto en el que la institucionalidad costarricense, a través de una reforma de la ley de Migración 2009 y cambios en la política institucional de la CCSS a partir del 2012, ha hecho un esfuerzo explícito por vincular el reconocimiento de derechos sociales y el acceso a servicios públicos para migrantes a su estado migratorio regular (Voorend, 2019), este artículo analiza el acceso real a servicios de salud de la población migrante nicaragüense en Costa Rica. Específicamente, nos preguntamos ¿qué tan determinante es el estado migratorio para el acceso de inmigrantes al seguro social y a los servicios de salud de la Caja Costarricense de Seguro Social (CCSS), institución emblemática del régimen de política social "excepcional" costarricense? 
Nos enfocamos en el sector de la salud porque, debido al principio de ius soli (modelo de ciudadanía de nacimiento), es ahí que la lucha por la inclusión en el estado costarricense es más obvia (Goldade, 2011). Además, en contraste con otros sectores (pensiones, educación), los servicios de salud se requieren a lo largo de la vida de una persona y, a diferencia de las transferencias familiares, se requiere en toda clase social. Asimismo, la incidencia de migrantes es más visible y su incorporación más polémica en este sector por la interacción cotidiana entre personas nacionales, migrantes y el estado que implica.

El aporte de este trabajo radica en que, en contraste con la mayoría de la literatura sobre la incorporación de inmigrantes en servicios sociales que se enfocan en términos de derechos formales y criterios de elegibilidad (Morissens y Sainsbury, 2005; Morissens, 2008; Voorend, 2019), nosotros analizamos el acceso real de inmigrantes a servicios de salud pública. Ello mediante el uso de técnicas cuantitativas, usando datos de una encuesta nacional y representativa entre inmigrantes nicaragüenses en Costa Rica, con un grupo de control costarricense.

Así, el artículo aporta al debate público sobre el reconocimiento formal y real de derechos sociales para inmigrantes y su incidencia en los servicios de salud pública. En un país cuyo estado social ha sido identificado reiteradamente como uno de los pocos con rasgos universalistas del Sur global, y cuyo peso de migrantes en el total de la población es el más alto en el contexto latinoamericano sirve como una especie de prueba de los esfuerzos para incorporar a las personas migrantes en los arreglos de bienestar universalistas de América Latina.

El artículo retoma la idea subyacente de que el universalismo no es un concepto dicotómico (Martínez Franzoni y Sánchez-Ancochea, 2013) y puede ser estratificado (Filguiera, 1998), analizando en qué medida diferentes grupos en la sociedad acceden a los servicios sociales de maneras distintas y enfocándonos en una de sus dimensiones más importantes: el acceso a servicios (Martínez Franzoni y Sánchez-Ancochea, 2013). En ese sentido se pretende subrayar la importancia de estudiar el déficit de implementación, definido por como la discrepancia entre derechos formales y su praxis.

En las siguientes páginas, primero se justifica la decisión de estudiar el caso de la salud pública en Costa Rica, evaluando la tensión que existe sobre la integración de migrantes en los servicios de salud. Después, se describe el proceso de la recolección de los datos 
primarios que sustenta la investigación, seguido por un análisis de contraste de hipótesis de medias y un análisis de regresiones probit para determinar la importancia del estado migratorio legal para distintos accesos a servicios de salud entre migrantes nicaragüenses. Por último, se presentan algunas reflexiones finales.

\section{MIGRACIÓN Y SERVICIOS DE SALUD EN COSTA RICA: UNA RELACIÓN TENSA}

Costa Rica presenta un caso interesante para estudiar la interacción entre la migración y la política social. Con alrededor de $9 \%$, es el país con el porcentaje de población migrante más alto de América Latina (Naciones Unidas, 2012; Voorend, 2019). Al mismo tiempo, el país tiene uno de los regímenes de política social más fuertes de la región (Martínez Franzoni, 2008). El seguro de salud universal, y los servicios de salud que provee la CCSS; institución que fue creada en 1941; son emblemáticos para este régimen. El acceso a los servicios de salud está mediado a través de un seguro de salud, cuyas tasas de cobertura son altas en comparación con otros países en el llamado Sur. El Seguro de Enfermedad y Maternidad (SEM), conocido como el seguro social, cubre hoy en día efectivamente alrededor del $87 \%$ de los casi 5 millones de costarricenses.

Dicho seguro, es requisito para acceder a los servicios de salud pública que no son de emergencia ${ }^{1}$, salvo para unas poblaciones bajo regímenes especiales de protección (ej. mujeres embarazadas, menores de dieciocho años). La afiliación puede ser directa, como seguro contributivo basado en impuestos sobre el salario (trabajo asalariado) o un seguro voluntario/independiente (trabajo no asalariado), o bien indirecta a través de un seguro familiar que permite la cobertura de familiares de personas contribuyentes con seguro directo.

Los servicios de salud costarricenses son de excepcional calidad (Martínez Franzoni y Sánchez-Ancochea, 2013). El país ha sido reconocido como una "historia de éxito" de salud, y un caso prometedor de "salud sin riqueza" (Noy, 2012). Junto con Cuba, Costa Rica lidera las expectativas de vida al nacer según las estimaciones

1 Los servicios de emergencia son disponibles siempre para cualquier persona que los necesite. Sin embargo, a las pacientes sin seguro social, se les presenta la factura del servicio posterior al tratamiento de emergencia. 
de población para el quinquenio del 2015 al 2020 (77.6 y 77.5 años respectivamente) en América (CEPAL, 2017), similar a países más avanzados como los Países Bajos (81.7 años) y Alemania (80.1 años) (Banco Mundial, 2017).

Sin embargo, las décadas de los 1980 y 1990 marcaron importantes cambios para el régimen de política social y el panorama migratorio. Dada la crisis de la deuda y las consecuentes políticas de austeridad, la política pública costarricense ha estado cada vez bajo más presión de hacer más con menos recursos per cápita (Martínez Franzoni y Sánchez-Ancochea, 2013). Como resultado, la base financiera para la política pública social se ha debilitado y la calidad de los servicios sociales deteriorado (idem).

En salud, los recortes en los suministros básicos fueron la esencia de las reformas de los años 80 y 90, que resultaron en aumentos en el tiempo y las listas de espera, y la insatisfacción de los pacientes (Martínez Franzoni y Sánchez-Ancochea, 2013). El gasto social público en salud per cápita, que casi se duplicó entre los inicios y finales de los 70 (de US $\$ 100$ a 200, medido en US\$ de 2000), disminuyó a principios de los años ochenta a unos US $\$ 120$ y estancó allí hasta la entrada la década de 2000, lo que, en términos reales, implicó una reducción. Entre 2000 y 2013, el número de médicos por cada mil habitantes ha disminuido de 1.33 a 1.11 (CEPAL, 2016) y hubo un auge en los servicios de salud privados (Martínez Franzoni y Sánchez-Ancochea, 2013), aumentos en las listas de espera para citas con el médico y una creciente insatisfacción con estos servicios (Dobles et al., 2013).

Justo cuando se empezó a sentir el deterioro de los servicios públicos y los recortes en la inversión pública en Costa Rica en los años 90, la inmigración alcanzó su punto máximo. Entre 1984 y 2000, la población inmigrante en Costa Rica creció a una tasa promedio anual de $7.5 \%$, la mayor parte explicada por la afluencia de nicaragüenses (INEC, 1984, 2000). En la década siguiente, la población migrante siguió creciendo (a 2.4\% anual) (INEC, 2000, 2011). Hoy, la población migrante representa aproximadamente el $9 \%$ de la población total, con el 6.7\% de origen nicaragüense (INEC, 2011).

Sin embargo, el deterioro en los servicios sociales se presenta, generalmente, "no como una consecuencia de las políticas neoliberales, sino como resultado de la migración de los nicaragüenses" (Sandoval, 2004, p. 444). Existe una percepción generalizada de que las personas migrantes nicaragüenses son culpables de la decadencia de los servicios sociales (Dobles et al., 2013; Goldade, 
2009; González y Varela, 2003), en particular el seguro social y la CCSS (Bonilla-Carrión, 2007; Goldade, 2009; Voorend, 2019). Como resultado, las voces de chovinismo del bienestar ${ }^{2}$ en el país abogan por reservar los servicios sociales para nacionales y la exclusión de inmigrantes, especialmente "ilegales".

La reacción del estado costarricense ha sido algo contradictoria. Por un lado, la última reforma de la Ley de Migración, en el 2009, justamente incluye un mayor reconocimiento de la importancia de la migración, y de los derechos de las personas migrantes (Fouratt, 2014). Específicamente, en contraste con leyes de otros países en la región, la ley hace múltiples referencias a los derechos humanos internacionales, comprometiendo al Estado explícitamente, por primera vez, con la integración de las personas inmigrantes en "los procesos económicos, científicos, políticos, sociales, laborales, educativos, culturales y deportivos" (Ley General de Migración N. ${ }^{\circ}$ 8764, art. 7) de la sociedad costarricense (Fouratt, 2014).

Por otro lado, en un esfuerzo explícito de limitar la inmigración irregular, el Estado ha creado mecanismos que han hecho más difícil el proceso de regularización y el acceso al seguro social, dos de los mecanismos claves para la integración de la población inmigrante (Voorend, 2019). Primero, para la regularización, la ley del 2009 establece que "todo trámite migratorio deba contemplar, como uno de sus requisitos básicos, contar con los seguros que brinda la Caja Costarricense de Seguro Social (CCSS)” (Ley N. ${ }^{\circ} 8764$, art. 7). Una motivación para esta medida fue recuperar "algunos de los recursos financieros que Costa Rica sentía que habían estado gastando en proporcionar servicios sociales, incluida la atención médica, a las poblaciones inmigrantes" (Clark, 2013, p. 132).

Sin embargo, los costos del proceso de regularización (estimados a sumar hasta USD 800) y la afiliación a la CCSS (9-15\% del ingreso estimado) son altos (IIS et al., 2012; Sandoval, 2012). Comparado con el salario mínimo de alrededor de USD 511, considerando que muchas personas inmigrantes ganan menos que esto (Voorend y

2 El chovinismo de bienestar representa el miedo entre los habitantes nativos de que los inmigrantes se apropien de los empleos y los servicios sociales (Faist, 1994), lo cual implica más restricciones en la política de bienestar, provocando la negación del acceso de los inmigrantes a las prestaciones sociales; así como limitaciones en la política migratoria. Lo anterior obstaculiza el derecho de los extranjeros a permanecer en el país y su acceso a programas sociales integrales (Banting, 2000). 
Robles, 2011), estos costos no solo no favorecen el proceso de regularización, sino implicarían que la ley "produciría la 'ilegalidad' que ella misma dice que procura erradicar" (Sandoval, 2012, p. 223).

Segundo, la interacción específica entre la política migratoria y la política social en Costa Rica provoca aún mayores obstáculos para la integración social de las personas inmigrantes. Siguiendo una serie de comunicaciones interinstitucionales entre la Dirección General de Migración y Extranjería (DGME) y la CCSS, este último (a solicitud del primero) establece que "las personas extranjeras que solicitan el aseguramiento para efectos de renovar su residencia deben presentar la cédula de residencia vigente" (CCSS, 2012a, p. 1). Esto se configura como una situación sin salida para muchas personas inmigrantes irregulares (Voorend, 2019): el seguro como requisito para la regularización, la regularización como requisito para el seguro.

Al mismo tiempo, la CCSS (2012b) establece que el seguro familiar para las personas inmigrantes puede cubrir solo a aquellos familiares que posean un estado migratorio regular. Es decir, cualquier familiar mayor de 18 años tiene que regularizar su estado migratorio primero, antes de disfrutar del seguro familiar. Para regularizar el estado migratorio es requisito contar con un seguro directo. En otras palabras, desde el año 2012, de facto, no existe un seguro familiar para personas inmigrantes. Esto crea condiciones desiguales importantes entre inmigrantes y nacionales: esta modalidad de seguro explica el $41 \%$ de la cobertura de la población costarricense (INEC, 2011).

Finalmente, estudios cualitativos sugieren que, a la hora de buscar servicios de salud, muchas personas migrantes enfrentan prácticas de la discriminación, incluso con un estado migratorio regular (Dobles et al., 2013; Fouratt, 2014; Fouratt y Voorend, 2018; SpesnyDos Santos, 2015). En la complicada estructura burocrática de la CCSS, el empleado de "ventanilla" puede tener una gran influencia sobre la concesión o negación de acceso de un migrante a los servicios de salud. Esto nos obliga ir más allá de los derechos formales, para estudiar el acceso real a los servicios sociales, como medida real de la integración social (Faist, 1994).

Todo esto da lugar a la sospecha de que el acceso a los servicios de salud pública está estratificado, primero, entre nacionales e inmigrantes, y segundo, entre migrantes con diferentes estados migratorios. En lo que sigue, se estudia con datos cuantitativos si esta sospecha tiene fundamento empírico. 


\section{METODOLOGÍA Y FUENTES DE DATOS}

\subsection{Datos}

Para estudiar la relación entre el estado migratorio y el acceso al seguro social y a los servicios de salud, se hizo, entre agosto y diciembre del 2013, una encuesta cuyo objetivo era recopilar datos primarios sobre los factores determinantes del acceso a los servicios sociales entre migrantes nicaragüenses en Costa Rica. Los datos, que se referenciarán como MISOC (2013), fueron recolectados con una muestra aleatoria entre 394 inmigrantes nicaragüenses, en diferentes partes del país, diseñada para ser representativa de las personas nacidas en Nicaragua que residen en Costa Rica ${ }^{3}$. Para promover las comparaciones, se incluyó una muestra de 401 costarricenses con características socioeconómicas similares ${ }^{4}$. MISOC (2013) complementa encuestas nacionales que solo capturan parcialmente información para migrantes y su acceso a los servicios públicos.

3 Siguiendo el estándar de las aplicaciones estadísticas, el diseño de la encuesta se construyó procurando obtener una potencia de 0.8 , un nivel de confianza del 95\% y un tamaño de efecto pequeño (d de Cohen) de 0.2. Con base en estos supuestos, el tamaño de muestra de inmigrantes nicaragüenses se calculó en $\mathrm{n}$ = 393 ("grupo de tratamiento"), y el mismo tamaño para nacionales ("grupo de control”). Una vez determinado el tamaño de la muestra, basado en condiciones prácticas como el presupuesto disponible, se decidió realizar la encuesta en 20 distritos y dentro de estos distritos en 50 Unidades de Muestreo Primario (UPMs - de entre aproximadamente 100-200 casas). En cada una de estas 50 UPM, se encuestó al azar a un total de 8 personas nacidas en Nicaragua y 8 nacidas en Costa Rica. Al final, se reunió información válida para 394 migrantes y 401 nacionales. Para asegurar la representatividad de la muestra nicaragüense, la selección de los distritos y las UPM se realizó utilizando la técnica de muestreo denominada Probabilidad Proporcional al Tamaño, con la probabilidad de seleccionar una unidad de muestreo (distritos) proporcional al tamaño de la población nacida en Nicaragua que reside en el distrito.

4 Para conseguir que el muestreo del grupo de control obtuviera individuos lo más similares posibles al grupo de inmigrantes en términos de rasgos observados y no observados, se seleccionaron aleatoriamente individuos nacidos en Costa Rica que viven en los mismos vecindarios que la población migrante. Dado que el muestreo se basó en áreas relativamente pequeñas que contenían alrededor de 100-200 casas, es probable que este enfoque dé lugar a que las poblaciones nacidas en Nicaragua y las nacidas en Costa Rica compartan características socioeconómicas relativamente similares. 


\subsubsection{Estadísticas descriptivas MISOC (2013)}

La Tabla 1 muestra una selección de estadísticas descriptivas de MISOC (2013). Se entrevistó a un número similar de hombres y mujeres de ambas nacionalidades. En línea con las expectativas, en promedio, los migrantes eran más jóvenes que los nacionales. Los hogares costarricenses y nicaragüenses están compuestos de una manera similar con respecto al número promedio de niños por hogar, así como a los niños menores de 6 años y dependientes del jefe del hogar. En promedio, los costarricenses tienen un año extra de educación. Asimismo, la mayoría de los nicaragüenses estudiaron en su país, y solo el $21.3 \%$ hizo uso del sistema educativo costarricense.

TABLA 1

ESTADÍSTICAS DESCRIPTIVAS DE MISOC 2013

\begin{tabular}{|c|c|c|c|}
\hline & \multirow{2}{*}{ Variable } & \multicolumn{2}{|c|}{ País de nacimiento } \\
\hline & & Costa Rica & Nicaragua \\
\hline $\mathrm{n}$ & & 401 & 394 \\
\hline \multirow{2}{*}{ Sexo } & Hombres entrevistados (\%) & 27.68 & 27.41 \\
\hline & Mujeres entrevistadas (\%) & 72.32 & 72.59 \\
\hline \multirow{2}{*}{ Edad } & Edad del entrevistado (promedio) & 45.85 & 39.78 \\
\hline & Desv. Estándar & 17.45 & 13.61 \\
\hline \multirow{8}{*}{ Hogar } & No. de niños (promedio) & 2.63 & 2.75 \\
\hline & Desv. Estándar. & 2.33 & 2.22 \\
\hline & No. de niños menores de 6 años (promedio) & 0.25 & 0.38 \\
\hline & Desv. Estándar. & 0.56 & 0.67 \\
\hline & No. de dependientes (promedio) & 2.39 & 2.91 \\
\hline & Desv. Estándar. & 1.94 & 1.95 \\
\hline & No. de personas que trabajan (promedio) & 1.56 & 1.69 \\
\hline & Desv. Estándar. & 0.93 & 0.99 \\
\hline \multirow{4}{*}{ Educación } & Años de educación (promedio) & 6.10 & 5.33 \\
\hline & Desv. Estándar. & 3.54 & 3.65 \\
\hline & País donde estudió - Costa Rica (\%) & 93.52 & 21.32 \\
\hline & País donde estudió - Nicaragua (\%) & 2.49 & 66.75 \\
\hline Trabajo & Personas con trabajo remunerado (\%) & 38.40 & 61.68 \\
\hline
\end{tabular}




\begin{tabular}{|c|c|c|c|}
\hline & \multirow{2}{*}{ Variable } & \multicolumn{2}{|c|}{ País de nacimiento } \\
\hline & & Costa Rica & Nicaragua \\
\hline \multirow{7}{*}{ Seguro social } & Directo: Trabajador asalariado & 19.0 & 18.8 \\
\hline & Directo: Independiente y voluntario & 11.2 & 10.9 \\
\hline & Directo: Pensionados & 13.2 & 2.3 \\
\hline & Indirecto: Asegurado por familiar & 32.2 & 24.1 \\
\hline & Directo: Asegurado por el Estado & 7.5 & 2.5 \\
\hline & Otro & 2.2 & 4.8 \\
\hline & No asegurado & 14.7 & 36.5 \\
\hline \multirow{4}{*}{ Antes de migrar } & Acceso a seguro social en in Nicaragua (\%) & & 16.8 \\
\hline & Trabajo pagado en Nicaragua (\%) & & 41.9 \\
\hline & Acceso a hospital (\%) & & 86.0 \\
\hline & Contacto en CR (\%) & & 69.0 \\
\hline \multirow{8}{*}{ Razón para migrar } & Falta de empleos en Nicaragua (\%) & & 50.1 \\
\hline & Diferencias en los salarios (\%) & & 47.7 \\
\hline & Mejor educación para los niños (\%) & & 37.6 \\
\hline & Necesidad de atención médica (\%) & & 4.7 \\
\hline & $\begin{array}{l}\text { Acceso familiar a servicios públicos (incluidos salud } \\
\text { y educación) (\%) }\end{array}$ & & 33.8 \\
\hline & Para la educación propia (\%) & & 13.7 \\
\hline & Reunificación familiar (\%) & & 30.5 \\
\hline & Razones políticas (\%) & & 19.8 \\
\hline \multirow{9}{*}{$\begin{array}{l}\text { Razón para elegir } \\
\text { Costa Rica como } \\
\text { destino }\end{array}$} & Contacto (familia/amigos) en CR (\%) & & 36.3 \\
\hline & Facilidad de tener trabajo remunerado (\%) & & 16.5 \\
\hline & Mejores pagos que en Nicaragua (\%) & & 6.9 \\
\hline & Proximidad (\%) & & 43.9 \\
\hline & Migración menos cara que a otros países (\%) & & 11.2 \\
\hline & Buenos servicios de salud/hospitales (\%) & & 3.3 \\
\hline & Embarazo o parto en CR (\%) & & 0.8 \\
\hline & Educación de los niños en CR (\%) & & 5.6 \\
\hline & Deportado en otro país (\%) & & 0.5 \\
\hline \multirow{6}{*}{$\begin{array}{l}\text { Estatus migratorio } \\
\text { en Costa Rica }\end{array}$} & Nacionalizado (\%) & & 6.9 \\
\hline & Residencia permanente (\%) & & 49.8 \\
\hline & Residencia temporal (\%) & & 5.8 \\
\hline & Irregular/“|legal” (\%) & & 19.8 \\
\hline & Visa de turismo $(\%)$ & & 8.9 \\
\hline & En proceso $(\%)$ & & 8.9 \\
\hline
\end{tabular}

Fuente: elaborado por los autores basado en MISOC (2013). 
Para los inmigrantes, la cobertura del seguro de salud es del 63.5\%, mientras el 36.5\% no cuentan con seguro (cf. INEC, 2011). Al mismo tiempo, el $14.7 \%$ de los nacionales no tenían seguro en 2013, ligeramente más alto que el $12.9 \%$ reportado por el último Censo Nacional (INEC, 2011).

Los datos de la encuesta confirman las tendencias generales con respecto al tipo específico de seguro de salud. Entre los costarricenses, el seguro directo es principalmente a través del trabajo asalariado (19.0\%) o seguro independiente/voluntario (11.2\%), como pensionistas (13.2\%), seguido por tasas mucho más bajas de seguro por parte del Estado (7.5\%). Es notable la importancia del seguro familiar para la cobertura de seguro de salud universalista de Costa Rica (32.2\%). Entre los migrantes, el seguro familiar es mucho menos común $(24.1 \%)$, mientras que las formas directas de seguro, como los trabajadores asalariados $(18.8 \%)$ y los trabajadores independientes $(10.9 \%)$ son similares a las tasas entre los nacionales. Solo unos pocos inmigrantes están asegurados por el estado $(2.5 \%)$.

En cuanto al proceso de migración, los encuestados nicaragüenses generalmente tenían poco acceso a la seguridad social en Nicaragua $(16.8 \%)$, y solo dos de cada cinco tenían un trabajo remunerado. Por el contrario, el $86 \%$ de los encuestados tenía acceso a servicios de salud (y hospitales) en Nicaragua. A las personas encuestadas se les pidió que enumeraran las tres razones principales para migrar. La falta de empleos en Nicaragua y la diferencia salarial entre Nicaragua y los posibles países de destino se mencionaron con mayor frecuencia (50.1 y 47.7\%, respectivamente). Alrededor de un tercio mencionó ambos motivos, y otro tercio mencionó al menos uno de ellos. Es decir, el $61 \%$ de la migración se explica por motivos laborales.

Otras razones fueron menos comunes, pero el futuro de los niños parece ser bastante importante: el 37.6\% mencionó la educación de los niños y el $33.8 \%$ el acceso de la familia a mejores servicios tales como salud y educación. Sin embargo, cuando se les preguntó por qué eligieron a Costa Rica como país de destino (y no a otro país), el acceso a la educación (5.6\%) o los hospitales (3.3\%) no parecía ser tan importante.

Por el contrario, la proximidad (43.9\%) y el consiguiente menor gasto de migración a Costa Rica (11.2\%) y no a un país más lejano, como los Estados Unidos, por ejemplo, parecen ser los principales impulsores de la migración. Además, las redes familiares o de amigos ya existentes en el país de destino (36.3\%) son importantes, así 
como los factores relacionados con el mercado laboral (23.4\%): la disponibilidad de puestos de trabajo $(16.5 \%)$ y los diferenciales salariales $(6.85 \%)$. Muchas personas migrantes viajan acompañadas $(58.1 \%)$, y aún más recibieron apoyo en Costa Rica (72.8\%), casi exclusivamente de amigos (92.5\%). Esto muestra la importancia de las redes en el proceso migratorio.

En Costa Rica, solo existían estimaciones anecdóticas e inexactas sobre la proporción exacta de migrantes irregulares, oscilando entre el 20 y el 40\% de la población migrante total (Karina Fonseca, Directora del Servicio Jesuita para Migrantes, Comunicación Personal, 5 de marzo de 2013). Los datos de MISOC (2013) sugieren que el 19.8\% de los nicaragüenses son irregulares o "ilegales", el 8.9\% tiene una visa de turista (que expira después de 3 meses) y otro $8.9 \%$ está en proceso de obtener documentos. Esta última condición, en principio, no debería impedir el acceso a los servicios sociales, pero en la práctica sí parece ser el caso (Fouratt, 2014). Según los datos de MISOC, el $62.4 \%$ tiene un estado migratorio regular (ciudadanía o residencia permanente o temporal). El otro $37.6 \%$ es "ilegal" o tiene un estado legal ambiguo que no permite el acceso a servicios de atención médica.

\subsection{Metodología}

En cuanto a la metodología para estudiar la importancia del estado migratorio para el acceso al seguro y a los servicios de salud, aplicamos pruebas de medias y regresiones multivariadas.

\subsubsection{Pruebas de medias}

Los análisis comparativos entre grupos buscan usualmente realizar cotejos entre muestras de dos poblaciones o más, para inferir si existen diferencias entre los mismos. En este caso, se utilizó la prueba estadística $t$ de student para llevar a cabo comparaciones de medias que evidencien las diferencias entre costarricenses y migrantes para diversas variables. Dichas pruebas fueron realizadas con un $5 \%$ de nivel de significancia.

\subsubsection{Análisis de regresiones}

Para evaluar cuantitativamente la importancia del estado migratorio para la integración en Costa Rica se estimaron regresiones 
para dos variables dependientes ${ }^{5}$ relevantes de las medidas de acceso al sistema de salud (SS): (1) la posesión de seguro de salud ${ }^{6}$ y (2) el acceso real a servicios de salud pública ${ }^{7}$. Las variables independientes utilizadas son vectores de: el proceso de migración $(M)$, características demográficas $(D)$, características de la inserción de una persona en el mercado laboral $(L M)$, características de los hogares $(H H)$ y otras dos variables $(C)$ : ubicación geográfica y calidad de la vivienda. La ecuación (I) representa esta relación:

$$
S S=f(M, D, L M, H H, C)
$$

Entre las variables independientes se encuentra un vector de características de migración (M). El modelo más básico incluye una variable dummy para los encuestados nacidos en Nicaragua para evaluar la importancia de la brecha nacional-migrante. La idea es examinar si existe alguna evidencia estadística de acceso desigual a seguros, atención médica y medicina basada en el origen nacional.

Se asume que el estado legal es clave para el acceso a servicios de salud. Por esto, las regresiones incluyen seis variables dummy que clasifican a los encuestados como nacionales costarricenses, migrantes nacionalizados, residentes (permisos de residencia temporal y permanente

5 Variables dependientes dicotómicas que toman un valor de 0 o 1 , donde el 1 significa tenencia de seguro o acceso a servicios de salud pública, según corresponda.

6 Las leyes y regulaciones de Costa Rica estipulan que la posesión del seguro de salud es una condición necesaria para acceder a los servicios de salud pública. La Ley de Migración estipula que el seguro de salud también es un requisito indispensable para el proceso de regularización. Por lo tanto, la primera variable dependiente que se analiza es si una persona tiene seguro de salud $(I)$.

7 Basado en estudios cualitativos (Fouratt, 2014; Fouratt y Voorend, 2018; Goldade, 2009; Spesny-Dos Santos, 2015) y el análisis de la política descrita en la sección anterior (cf. Voorend, 2019), tenemos la sospecha de que puede haber una brecha entre el tener un seguro de salud y el acceso real a servicios de salud, y que esta brecha se da por la condición de migrante independientemente del estado migratorio particular. Por lo tanto, en un segundo conjunto de regresiones se usa el acceso real a los servicios de salud pública $(\mathrm{H})$ como variable dependiente. A los encuestados se les preguntó sobre el tipo de servicios de salud y medicina que buscan cuando lo necesitan. Específicamente, se preguntó si buscarían los servicios de atención médica de CCSS cuando necesiten dichos servicios. Por lo tanto, el acceso a la atención médica y a la medicina pública se refiere a las percepciones de acceso a los servicios de CCSS, en parte en función de las experiencias previas de los encuestados con dicho acceso. 
y permisos de trabajo), migrantes con una visa de turista que aún no había expirado en el momento de la entrevista), los migrantes que tienen su documentación para cualquier estado migratorio regular en proceso, y los migrantes "ilegales" (sin estatus migratorio legal).

Se espera que las categorías de migrantes nacionalizados y residentes legales se correlacionen positivamente con el acceso a salud, aunque no debe ser diferente al de los nacionales costarricenses. Si, utilizando a los nacionales como base para comparar y controlar otros factores, cualquiera de estas variables está negativamente correlacionado con el seguro o la atención médica, puede interpretarse como evidencia de que alguna característica intrínseca de ser nicaragüense inhibe el acceso. Si, por el contrario, la ciudadanía se correlaciona positivamente con cualquiera de las variables dependientes, esto sugiere que los requisitos legales para obtener un seguro se traducen en mayores tasas de seguro entre una de dos opciones:

1. denizens

2. inmigrantes regulares

Para los "ilegales" y los turistas, se espera una fuerte relación negativa, ya que estas categorías, en principio, excluyen el acceso al seguro de salud pública y la asistencia sanitaria. Finalmente, la ley prescribe que una vez que la documentación está en proceso para un estado migratorio regular, un migrante puede obtener un seguro de salud (temporal y condicional) de la CCSS mostrando un recibo para demostrar que las condiciones requeridas por la DGME se han cumplido. Es decir, esta categoría no debería correlacionarse negativamente con el seguro.

Además, siguiendo la revisión de literatura, se espera que la exposición temporal en la sociedad de acogida, la exposición geográfica y la exposición social (años desde la primera migración a Costa Rica) (Danzer y Ulku, 2008) incidan en la integración de migrantes en general y en la obtención de seguros de salud y acceso a servicios en particular. La exposición social se aproximó mediante un proxy de redes sobre si conocía a alguien en el país antes de migrar. La exposición geográfica fue capturada por una variable de control para zona (rural o urbana), donde no se espera que tenga ningún impacto en el seguro de salud o el acceso a servicios, dada la cobertura universal del sistema de salud de Costa Rica.

Otras variables operacionalizan características demográficas (D) (edad y sexo), características del mercado laboral (LM) (formalidad del empleo capturada por categorías laborales basadas en códigos ISCO de la OIT y variables dummy de derechos laborales, educación formal en 
años e ingresos de los entrevistados como variable categórica), características del hogar (sexo del jefe del hogar, tipología de tipos de familia, presencia de niños entre 0 y 6 y de 6 y 18 años) y una variable de la calidad de la vivienda como indicador del estado socioeconómico.

Se espera que las mujeres tengan menos acceso al seguro y acceso a los servicios de salud, mientras que la formalidad de los empleos se correlacione positivamente con ambas variables dependientes, al igual que la educación y los ingresos. Se espera que la presencia de niños, especialmente los más jóvenes (entre 0 y 6 años) se correlacione positivamente con el seguro de salud y el acceso a servicios, porque son un grupo protegido en Costa Rica y siempre deben ser atendidos en el sistema médico, independientemente de la nacionalidad y estado migratorio. La investigación cualitativa ha sugerido que los niños pueden servir como catalizadores para el acceso de adultos (Fouratt y Voorend, 2018).

\section{Modelos de regresión}

Dada la naturaleza de las variables dependientes, se utilizan modelos de regresión probit utilizando como método de estimación la máxima verosimilitud. La ecuación 2 muestra que la probabilidad de acceso al sistema de salud (SS, medida como posesión de seguro de salud - $I$-, o acceso a servicios de salud - $H$ ), se trata como una función de la migración $(\mathrm{M})$, demográfica $(D)$, características del mercado de trabajo $(L M)$ y del hogar $(H H)$ y las otras dos variables de control $(C)$.

$$
\operatorname{Pr}\left[S S_{1}=1\right]=\operatorname{Prob}\left[\beta_{L M} L M_{i}+\beta_{D} D_{i}+\beta_{H H} H H_{i}+\beta_{M} M_{i}+\beta_{C} C_{i}+\varepsilon_{i}>0\right]
$$

Se especificaron cuatro modelos diferentes para el análisis de regresión con la muestra completa (nacionales e inmigrantes). Para verificar la solidez de las estimaciones, los cuatro modelos van desde especificaciones simples hasta más completas. Por ejemplo, el modelo 1 solo incluye la variable dummy "ilegal" como medida para el estado migratorio. En los modelos subsiguientes, el estado migratorio se desglosa aún más para incluir los otros estados legales diferentes. El modelo final, que solo se ejecuta para el acceso a los servicios de salud pública, incluye un seguro de salud como variable independiente para confirmar su importancia para garantizar el acceso a la atención médica pública. Si las variables de estado migratorio siguen siendo significativas después de controlar el estado del seguro, implica que las categorías migratorias también influyen en el acceso. 


\section{RESULTADOS}

Después de analizar algunos estadísticos descriptivos de MISOC (2013), se presentan los resultados de los análisis descritos previamente para investigar si existen diferencias entre el acceso a la salud pública entre población migrante nicaragüense y costarricense.

\subsection{Acceso a servicios de salud}

Uno de los resultados esperados es que el estado migratorio condiciona el acceso a los servicios de salud pública. En la Tabla 2 se reportan el tipo de atención médica y medicina que usan las personas entrevistadas. En especial, se preguntó si ellos usarían los servicios de salud y medicina de la CCSS en caso de ser necesario, es decir los datos hacen referencia a las percepciones de las personas encuestadas de su acceso a los servicios a la CCSS.

TABLA 2

ACCESO A LA ATENCIÓN SANITARIA Y MEDICINA PÚBLICA PARA NACIONALES E INMIGRANTES NICARAGÜENSES SEGÚN ESTADO MIGRATORIO, 2013

(Porcentajes)

\begin{tabular}{|l|c|c|c|c|c|c|c|}
\hline \multicolumn{1}{|c|}{ Variable } & $\begin{array}{c}\text { Costa } \\
\text { Rica }\end{array}$ & \multicolumn{7}{c|}{ Nicaragua } \\
\hline & Total & Total & $\begin{array}{c}\text { Naciona- } \\
\text { lizados }\end{array}$ & Residentes & "Ilegales" & Turistas & $\begin{array}{c}\text { En } \\
\text { proceso }\end{array}$ \\
\hline $\mathrm{n}$ & 401 & 394 & 27 & 219 & 78 & 35 & 35 \\
\hline $\begin{array}{l}\text { CCSS. Acceso gratuito } \\
\text { de atención médica }\end{array}$ & 78.8 & 57.9 & 74.1 & 77.2 & 26.9 & 25.7 & 25.7 \\
\hline $\begin{array}{l}\text { CCSS. Sin acceso gratuito } \\
\text { de atención médica }\end{array}$ & 21.2 & 42.1 & 25.9 & 22.8 & 73.1 & 74.3 & 74.3 \\
\hline $\begin{array}{l}\text { CCSS Asistencia } \\
\text { médica pagada }\end{array}$ & 1.5 & 5.8 & 0.0 & 2.7 & 7.7 & 25.7 & 5.7 \\
\hline No son atendidos & 2.0 & 18.5 & 3.7 & 6.4 & 39.7 & 25.7 & 51.4 \\
\hline $\begin{array}{l}\text { Preferencia por atención } \\
\text { médica privada }\end{array}$ & 7.0 & 4.1 & 3.7 & 3.7 & 6.4 & 2.9 & 2.9 \\
\hline Otras razones & 4.5 & 6.9 & 3.7 & 5.0 & 11.5 & 11.4 & 5.7 \\
\hline Razones desconocidas & 6.2 & 6.9 & 14.8 & 5.0 & 7.7 & 8.6 & 8.6 \\
\hline Total & 100 & 100 & 100 & 100 & 100 & 100 & 100 \\
\hline
\end{tabular}

Fuente:elaborado por los autores basado en MISOC (2013). 
En particular, el acceso de los costarricenses a los servicios de salud pública es más frecuente que el de los nicaragüenses (78.8 frente a un 57.9\%). La notable diferencia entre costarricenses e inmigrantes se debe a los más altos índices de encuestados inmigrantes no asegurados (36.5\%) en comparación con nacionales (14.7\%). Ahora bien, si sólo los no asegurados son los que no tuvieran derecho a la atención médica pública, un $85.3 \%$ y $63.5 \%$ debería tener acceso a los servicios de la CCSS. Lo cual quiere decir que, existe un margen promedio de un 6.5 y un 5.4 puntos porcentuales constituido por personas aseguradas sin acceso a los servicios de salud pública. En un 18.5\% de los casos, los nicaragüenses expresaron que no podrían recibir atención en la CCSS debido a su situación legal, mientras que, en el caso de los costarricenses, la razón más usual por la que no asisten a la CCSS es porque prefieren el servicio de salud privado $(7 \%)$.

Los datos señalan una división evidente entre los denizens (migrantes nacionalizados o residentes) y las otras categorías de estado migratorio para el acceso a los servicios de salud. Entre los inmigrantes con estado "ilegal" y turístico y aquéllos con su documentación en trámite, solo uno de cada cuatro tiene acceso a los servicios de salud de la CCSS, en comparación con tres de cada cuatro denizens. El acceso es particularmente bajo para aquellos inmigrantes en proceso de regularización de documentos, tomando en cuenta que, en principio, su condición no debería de inhibirles el acceso al seguro y los servicios médicos. En contraste, los denizens y ciudadanos costarricenses poseen un acceso a la salud a escalas muy similares, lo cual sugiere que el estado migratorio legal efectivamente favorece la integración de los inmigrantes.

\subsection{Pruebas de medias}

Con el fin de comprobar si las diferencias entre nacionales e inmigrantes, y entre inmigrantes "ilegales" y denizens (residentes e inmigrantes nacionalizados) son relevantes a nivel estadístico, se muestran los resultados del análisis de pruebas de medias. La Tabla 6 presenta una selección de variables relacionadas con el acceso a la atención médica y la medicina pública, y otras variables que miden algunas características generales, tales como edad, o características vinculadas al proceso migratorio. 
Primero, en promedio los nacionales son seis años mayores que los inmigrantes y los denizens casi 4 años mayores que inmigrantes "ilegales". Además, en promedio, los nacionales han tenido más acceso a la educación formal que los inmigrantes (0.8 años). Sin embargo, no existe una diferencia significativa entre los inmigrantes con diferentes estados migratorios. En cuanto la categoría de ingresos, considerando la población trabajadora, no existen diferencias significativas entre los nacionales, denizens e inmigrantes "ilegales", lo cual refleja nuevamente que se seleccionó de forma efectiva un grupo control costarricense con condiciones socioeconómicas similares a la población inmigrante nicaragüense.

TABLA 3

PRUEBAS DE MEDIAS PARA LAS VARIABLES SELECCIONADAS SEGÚN PAÍS DE ORIGEN Y ESTADO MIGRATORIO, 2013

\begin{tabular}{|c|c|c|c|c|c|c|c|c|}
\hline \multicolumn{2}{|r|}{ Variables } & \multicolumn{4}{|c|}{ Promedios } & \multicolumn{3}{|c|}{ Valores $p$} \\
\hline & & $\begin{array}{c}\text { Costa } \\
\text { Rica }\end{array}$ & Nicaragua & Denizen & 'llegal' & $\begin{array}{l}\text { Dif. CR- } \\
\text { NIC }\end{array}$ & $\begin{array}{l}\text { Dif. CR- } \\
\text { Denizen }\end{array}$ & $\begin{array}{l}\text { Dif. Denizen } \\
\text { - 'llegal' }\end{array}$ \\
\hline \multirow{3}{*}{ Características1/ } & Edad & 45.85 & 39.78 & 42.13 & 35.87 & $6.07^{\star \star \star}$ & $3.72^{\star \star}$ & $6.25^{\star \star \star}$ \\
\hline & Años de escolaridad & 6.11 & 5.33 & 5.26 & 5.45 & $0.77^{\star \star}$ & $0.85^{\star \star}$ & -0.20 \\
\hline & Categoría de ingresos & 1.61 & 2.14 & 2.35 & 1.78 & $-0.53^{\star \star}$ & $-0.75^{\star \star \star}$ & $0.58^{\star}$ \\
\hline \multirow{9}{*}{$\begin{array}{l}\text { Servicios de } \\
\text { Salud }\end{array}$} & $\begin{array}{l}\text { CCSS Salud pública } \\
\text { gratuita }\end{array}$ & 0.79 & 0.58 & 0.77 & 0.26 & $0.21^{\star \star \star}$ & 0.06 & $0.51^{\star \star \star}$ \\
\hline & $\begin{array}{l}\text { CCSS Salud pública } \\
\text { gratuita para niños } \\
\text { (menores de 18) }\end{array}$ & 0.45 & 0.60 & 0.63 & 0.56 & $-0.15^{\star \star \star}$ & $-0.18^{\star \star \star}$ & 0.07 \\
\hline & Seguro de salud & 0.85 & 0.64 & 0.87 & 0.24 & $0.22^{\star \star \star}$ & -0.02 & $0.63^{\star \star \star}$ \\
\hline & Trabajadores asalariados & 0.19 & 0.19 & 0.25 & 0.09 & 0.00 & -0.06 & $0.16^{\star \star \star}$ \\
\hline & \begin{tabular}{|l|} 
Trabajadores \\
independientes y \\
voluntarios
\end{tabular} & 0.11 & 0.11 & 0.16 & 0.02 & 0.00 & -0.05 & $0.14^{\star \star \star}$ \\
\hline & Seguro familiar & 0.32 & 0.24 & 0.35 & 0.06 & $0.08^{\star}$ & -0.03 & $0.29^{\star \star \star}$ \\
\hline & Seguro por el Estado & 0.08 & 0.03 & 0.03 & 0.01 & $0.05^{\star \star}$ & 0.04 & 0.02 \\
\hline & Pensionados del RNC e IVM & 0.13 & 0.02 & 0.03 & 0.01 & $0.11^{\star \star \star}$ & $0.10^{\star \star \star}$ & 0.01 \\
\hline & Otros & 0.01 & 0.01 & 0.00 & 0.01 & 0.01 & 0.01 & -0.00 \\
\hline
\end{tabular}

$1 /$ Las características son de la persona entrevistada

${ }^{*} p<0.05 .{ }^{* \star} p<0.01$. ${ }^{\star \star *} p<0.001$

Fuente: elaborado por los autores basado en MISOC (2013). 
Segundo, no hay diferencias significativas entre denizens e inmigrantes "ilegales" que se relacionen con las variables de migración, salvo que las personas inmigrantes con estado migratorio regular hayan estado en el país en promedio siete años más que las personas inmigrantes "ilegales". Esto respalda el argumento de que el mayor tiempo de exposición a la sociedad receptora podría representar un factor importante para la integración.

Con respecto a la salud pública, el análisis de medias confirma que los nacionales e inmigrantes tienen un acceso diferenciado al seguro y los servicios de salud. Para el seguro de salud (sin hacer un desglose del tipo), inmigrantes tienen menos acceso que nacionales, e "ilegales" tienen menos acceso que denizens. Sin embargo, en consonancia con los resultados anteriores, el seguro de trabajadores asalariados o asegurados independientes o voluntarios no varía significativamente entre nacionales e inmigrantes. Para todos los demás tipos de seguro, las personas inmigrantes tienen peor acceso al seguro. Especialmente el seguro familiar, el cual es de suma importancia para la cobertura del seguro social en Costa Rica. Además, las personas inmigrantes "ilegales" tienen inferior acceso a los seguros como trabajadores asalariados, seguros independientes o voluntarios y seguros familiares que los denizens.

El análisis de medias comprueba que los denizens y los nacionales tienen índices muy parecidos de acceso a la salud pública. De hecho, los denizens sobrepasan a los nacionales por un 17.7 puntos porcentuales cuando se trata del acceso a la salud pública para sus hijos. Por otra parte, aunque factores tales como la edad, la educación y los ingresos sean significativamente diferentes, no hay pruebas estadísticas que confirmen las diferencias en el acceso a la atención médica pública entre los denizens y los costarricenses.

\subsection{Regresiones}

Los resultados del análisis de regresión se presentan en la Tabla 4. En la misma se muestran los efectos marginales basados en el análisis de regresión probit con la variable dependiente tenencia de 
TABLA 4

ESTIMACIONES OBTENIDAS DE LAS REGRESIONES PROBIT

PARA LAS VARIABLES DEPENDIENTES: TENENCIA DE

SEGURO SOCIAL (I) Y ACCESO A SERVICIOS DE SALUD PÚBLICOS $(\mathrm{H})$

(Efectos marginales reportados)

\begin{tabular}{|c|c|c|c|c|c|c|c|c|c|}
\hline \multirow{2}{*}{\multicolumn{2}{|c|}{$\begin{array}{c}\text { Variable dependiente } \\
\text { No. de regresión }\end{array}$}} & \multicolumn{4}{|c|}{ Acceso a seguro social (I) } & \multicolumn{4}{|c|}{ Acceso a servicios de salud públicos (H) } \\
\hline & & 1 & 2 & 3 & 4 & 5 & 6 & 7 & 8 \\
\hline \multirow{4}{*}{ D } & Edad & $0.013^{*}$ & $0.012^{*}$ & 0.006 & 0.007 & 0.009 & 0.003 & 0.005 & -0.001 \\
\hline & & $(0.006)$ & $(0.006)$ & $(0.007)$ & $(0.007)$ & $(0.007)$ & $(0.007)$ & $(0.007)$ & $(0.007)$ \\
\hline & Sexo & $-0.157^{\star \star \star}$ & $-0.142^{* *}$ & $-0.164^{\star *}$ & $-0.164^{\star \star}$ & -0.049 & -0.065 & -0.058 & 0.002 \\
\hline & & $(0.046)$ & $(0.046)$ & $(0.055)$ & $(0.055)$ & $(0.047)$ & $(0.056)$ & $(0.056)$ & $(0.058)$ \\
\hline \multirow{19}{*}{ M } & Nicaragüense & -0.074 & & & & & & & \\
\hline & & $(0.056)$ & & & & & & & \\
\hline & Años en CR & $0.006^{\star \star}$ & $0.006^{\star \star \star}$ & 0.002 & 0.002 & $0.006^{\star \star \star}$ & 0.003 & 0.003 & 0.003 \\
\hline & & $(0.002)$ & $(0.001)$ & $(0.002)$ & $(0.002)$ & $(0.001)$ & $(0.002)$ & $(0.002)$ & $(0.002)$ \\
\hline & Contacto en CR & -0.001 & -0.007 & 0.014 & 0.017 & -0.016 & 0.008 & 0.013 & 0.011 \\
\hline & & $(0.041)$ & $(0.041)$ & $(0.050)$ & $(0.051)$ & $(0.048)$ & $(0.056)$ & $(0.056)$ & $(0.056)$ \\
\hline & & & - & - & - & - & - & - & $-0.219^{*}$ \\
\hline & "Illegal" & & $0.438^{\star \star \star *}$ & $-0.510^{\star \star *}$ & $-0.495^{\star \star \star}$ & $-0.369^{\star \star \star}$ & $-0.433^{\star \star \star}$ & $-0.427^{\star \star \star}$ & \\
\hline & & & $(0.067)$ & $(0.094)$ & $(0.095)$ & $(0.064)$ & $(0.082)$ & $(0.083)$ & $(0.096)$ \\
\hline & Nacionalizado & & & $0.149^{\star *}$ & $0.145^{\star \star}$ & & -0.017 & -0.021 & -0.099 \\
\hline & & & & $(0.048)$ & $(0.045)$ & & $(0.103)$ & $(0.103)$ & $(0.112)$ \\
\hline & Residencia & & & 0.047 & 0.062 & & 0.057 & 0.064 & 0.024 \\
\hline & & & & $(0.058)$ & $(0.055)$ & & $(0.064)$ & $(0.064)$ & $(0.072)$ \\
\hline & & & & - & - & & - & - & -0.150 \\
\hline & Visa de turismo & & & $0.637^{\star \star \star}$ & $0.658^{\star \star \star}$ & & $0.435^{\star \star \star}$ & $0.442^{\star \star \star}$ & \\
\hline & & & & $(0.111)$ & $(0.104)$ & & $(0.106)$ & $(0.105)$ & $(0.133)$ \\
\hline & & & & $-0.386^{\star \star}$ & $-0.390^{\star \star}$ & & - & - & $-0.318^{*}$ \\
\hline & En proceso & & & & & & $0.451^{\star \star \star}$ & $0.450^{\star \star \star}$ & \\
\hline & & & & $(0.123)$ & $(0.122)$ & & $(0.098)$ & $(0.098)$ & $(0.129)$ \\
\hline
\end{tabular}


De derechos a hechos. El acceso diferenciado entre nacionales y migrantes... 141

\begin{tabular}{|c|c|c|c|c|c|c|c|c|c|}
\hline \multirow{2}{*}{\multicolumn{2}{|c|}{$\begin{array}{c}\text { Variable dependiente } \\
\text { No. de regresión }\end{array}$}} & \multicolumn{4}{|c|}{ Acceso a seguro social (I) } & \multicolumn{4}{|c|}{ Acceso a servicios de salud públicos (H) } \\
\hline & & \multirow[t]{2}{*}{1} & \multirow[t]{2}{*}{2} & \multirow[t]{2}{*}{3} & \multirow[t]{2}{*}{4} & \multirow[t]{2}{*}{5} & \multirow[t]{2}{*}{6} & \multirow[t]{2}{*}{7} & \multirow{2}{*}{$\frac{8}{0.552^{* k+}}$} \\
\hline \multirow{16}{*}{$L M$} & Seguro de salud & & & & & & & & \\
\hline & & & & & & & & & $(0.047)$ \\
\hline & Aguinaldo & -0.058 & -0.076 & -0.055 & -0.038 & -0.049 & -0.013 & -0.014 & -0.006 \\
\hline & & $(0.093)$ & $(0.095)$ & $(0.103)$ & $(0.104)$ & $(0.095)$ & $(0.099)$ & $(0.099)$ & $(0.096)$ \\
\hline & Incapacidades & -0.055 & -0.065 & -0.081 & -0.073 & -0.062 & -0.059 & -0.052 & -0.023 \\
\hline & & $(0.087)$ & $(0.088)$ & $(0.086)$ & $(0.085)$ & $(0.086)$ & $(0.088)$ & $(0.087)$ & $(0.083)$ \\
\hline & Vacaciones pagas & 0.112 & 0.115 & 0.083 & 0.087 & 0.109 & 0.054 & 0.052 & 0.034 \\
\hline & & $(0.075)$ & $(0.074)$ & $(0.081)$ & $(0.082)$ & $(0.086)$ & $(0.097)$ & $(0.095)$ & $(0.093)$ \\
\hline & Seguro de riesgo & $0.243^{\star * \star}$ & $0.250^{\star \star \star}$ & $0.237^{\star \star \star}$ & $0.229^{\star \star \star}$ & $0.164^{\star \star}$ & $0.158^{*}$ & $0.143^{*}$ & -0.005 \\
\hline & & $(0.033)$ & $(0.032)$ & $(0.035)$ & $(0.034)$ & $(0.060)$ & $(0.065)$ & $(0.067)$ & $(0.077)$ \\
\hline & Pago de horas extra & -0.021 & -0.018 & -0.012 & -0.022 & 0.039 & 0.049 & 0.047 & 0.078 \\
\hline & & $(0.068)$ & $(0.069)$ & $(0.071)$ & $(0.068)$ & $(0.063)$ & $(0.063)$ & $(0.065)$ & $(0.063)$ \\
\hline & Educación & 0.001 & -0.001 & -0.002 & -0.002 & -0.001 & -0.001 & -0.000 & 0.001 \\
\hline & & $(0.005)$ & $(0.005)$ & $(0.005)$ & $(0.005)$ & $(0.006)$ & $(0.006)$ & $(0.006)$ & $(0.006)$ \\
\hline & Categoría de ingreso & 0.008 & 0.006 & $0.020+$ & $0.026^{*}$ & $-0.028^{\star \star}$ & -0.014 & -0.012 & -0.022 \\
\hline & & $(0.009)$ & $(0.009)$ & $(0.011)$ & $(0.012)$ & $(0.010)$ & $(0.014)$ & $(0.014)$ & $(0.014)$ \\
\hline \multirow{2}{*}{ HH } & Hijos menores de 6 & 0.012 & -0.018 & -0.019 & -0.017 & 0.055 & 0.054 & 0.054 & $0.083+$ \\
\hline & & $(0.040)$ & $(0.042)$ & $(0.042)$ & $(0.042)$ & $(0.047)$ & $(0.048)$ & 10.047 & $(0.049)$ \\
\hline \multirow{4}{*}{ c } & Zona urbana & 0.007 & -0.014 & 0.001 & 0.027 & -0.007 & 0.054 & 0.060 & 0.061 \\
\hline & & $(0.033)$ & $(0.034)$ & $(0.036)$ & $(0.039)$ & $(0.039)$ & $(0.043)$ & $(0.045)$ & $(0.046)$ \\
\hline & Calidad de la casa & $0.011^{\star}$ & $0.010+$ & 0.008 & 0.007 & $0.014^{*}$ & $0.013^{*}$ & $0.013^{\star}$ & $0.012+$ \\
\hline & & $(0.005)$ & $(0.005)$ & $(0.006)$ & $(0.006)$ & $(0.006)$ & $(0.006)$ & $(0.006)$ & $(0.006)$ \\
\hline Observaciones & & 795 & 795 & 795 & 795 & 795 & 795 & 795 & 795 \\
\hline Pseudo R² & & 0.221 & 0.271 & 0.357 & 0.375 & 0.135 & 0.200 & 0.209 & 0.325 \\
\hline Log Verosimilitud & & -329.18 & -329.18 & -290.39 & -282.30 & -428.2 & -395.95 & -391.62 & -333.94 \\
\hline
\end{tabular}

Errores estándar robustos entre paréntesis: ${ }^{\star \star *} p<0.001,{ }^{\star \star} p<0.01,{ }^{*} p<0.05,+p<0.1$

Fuente: elaborado por los autores basado en MISOC (2013). 
un seguro de salud (I) para las columnas 1-4 y acceso a los servicios de salud pública $(\mathrm{H})$ en las columnas $5-8^{8}$.

De las variables independientes en el vector de migración (M), las más importantes son las relacionadas con el estado migratorio. Existe una fuerte evidencia estadística de que las variables de estado migratorio son determinantes importantes en la probabilidad de estar asegurado. Las categorías 'ilegal' y 'turística' son consistentemente significativas y tienen grandes efectos. Los migrantes "ilegales" tienen entre 44 y 51 puntos porcentuales menos probabilidades de estar asegurados en comparación con los nacionales (regresiones 1-4). El efecto es aún más fuerte para los nicaragüenses en el país con estatus de turista (visa turista). En promedio, de acuerdo con las expectativas, ser un turista reduce la probabilidad de seguro en aproximadamente 65 puntos porcentuales.

En principio, aunque se espera que la condición de migrante nacionalizado esté correlacionado positivamente con el acceso a seguro social (I), no debería ser significativo en estas regresiones, ya que la base son los nacionales costarricenses. Es decir, en principio, no hay ninguna razón para suponer que un migrante residente o nacionalizado tenga mejor o peor acceso al seguro de salud que un nacional. En consecuencia, los residentes no muestran una diferencia significativa, pero en las regresiones 3 y 4, los migrantes nacionalizados parecen tener mayores probabilidades de estar asegurados que los nacionales costarricenses en alrededor de 15 puntos porcentuales. Asimismo, la categoría 'en proceso' es significativa y está correlacionada negativamente con el seguro de salud; el efecto es bastante grande en alrededor de 40-60 puntos porcentuales, por tanto, los resultados muestran que los migrantes cuyo papeleo está en proceso de regularización no están asegurados. Por otro lado, este conjunto de regresiones (I) sugieren que las mujeres poseen menor probabilidad de acceder a seguro social (con efectos negativos entre 14 y 16 puntos). Esto coincide con investigaciones cualitativas que sugieren que las mujeres son típicamente las últimas de asegurarse en una familia (Fouratt y Voorend, 2018; Spesny-Dos Santos, 2015) por su inserción

8 Sobre el ajuste de los modelos, cabe destacar que, tomando en cuenta que los mismos pretender modelar comportamientos humanos, los pseudo R2 indican que hay un ajuste bueno de los mismos. El R2 aumenta cuando se incorporan las variables del estatus migratorio de los individuos de la muestra (tendencia que se mantiene en ambos conjuntos de regresiones según variable respuesta), demostrando la relevancia de este factor en el proceso de integración de los migrantes. 
informal, o, como también se puede apreciar en nuestra muestra, de mayor inserción en labores domésticas, que suelen caracterizarse por la carencia de aseguramiento por parte de sus patrones.

Las regresiones 5 a 8 estiman los efectos relacionados con el acceso a los servicios de salud pública (H). Nuevamente, el estado migratorio es clave para el acceso a los servicios de salud. La "ilegalidad" es un determinante importante del acceso público a la asistencia sanitaria, especialmente a través de la relación negativa con el seguro de salud. Si bien su magnitud es grande después de controlar por la tenencia del seguro (regresión 8), la "ilegalidad" continúa ejerciendo un efecto negativo en el acceso a la atención médica. Por el contrario, el efecto negativo para los turistas se ve disminuido y se convierte en (casi) insignificante al controlar por la tenencia de seguro. En general, esto sugiere que el acceso a los servicios de salud depende ante todo del seguro, y como se vio antes, el seguro depende del estado migratorio. Como se esperaba, las categorías de ciudadanía no muestran diferencias significativas en comparación con los migrantes nacionalizados. Finalmente, los migrantes con su documentación en proceso tienen consistentes y significativamente menores probabilidades de acceso a la atención médica, aunque técnicamente esto no debería ser el caso.

Entre las variables que capturan las características demográficas (D), existe evidencia estadística limitada de que la edad se correlaciona con la probabilidad de tener seguro. En las regresiones 1 y 2, la edad se correlaciona positivamente con el acceso a seguro social (I). Entre los migrantes y la muestra total, los resultados sugieren que cada año aumenta la probabilidad de tener un seguro en 0,8 y 0,6 puntos porcentuales, respectivamente. En otras palabras, con base en las estimaciones para la muestra total, en igualdad de condiciones, una persona de 40 años tiene 6 puntos porcentuales más de probabilidades de estar asegurado que su contraparte más joven de 10 años. Si bien esto es consistente con las expectativas iniciales, los resultados no son consistentes con respecto a las regresiones, ya que esa correlación se silencia cuando se controlan otras variables en los modelos más completos. En todas las especificaciones, ser hombre se asocia con una probabilidad de 14 a 16 puntos porcentuales menor de estar asegurado en comparación con las mujeres. En contraste, para acceder a los servicios de salud $(\mathrm{H})$, no hay evidencia estadística de que la edad o el sexo afecten la probabilidad de acceder a la atención médica. 
Por otro lado, las estimaciones obtenidas apuntan a que tener un contacto con Costa Rica antes de migrar no afecta la probabilidad de tener un seguro ni acceder a los servicios de salud pública. De manera similar, el tiempo de exposición a la sociedad de acogida, solo es significativo en los modelos más básicos, pero el efecto desaparece cuando se incluyen otras variables. Las regresiones 1, 2 y 5 sugieren que un migrante con 20 años en Costa Rica tiene una probabilidad $6 \%$ mayor de tener un seguro (o tener acceso a servicios de salud) que un migrante con 10 años en Costa Rica. El efecto positivo es consistente con la teoría, pero la evidencia es débil. Es decir, en general, parece que el tiempo que se pasa en Costa Rica no tiene mucha influencia en el acceso a los seguros de salud y los servicios de salud.

Las variables dummy de derechos laborales muestran resultados relevantes. Mientras que las vacaciones pagadas son insignificantes en todas las regresiones, los otros derechos laborales muestran significancia en al menos una de las regresiones. En ese sentido, el seguro de riesgo es consistentemente significativo y muestra una correlación positiva con $I$. Entre los migrantes, tener un seguro de riesgo aumenta la probabilidad de tener también un seguro de salud en aproximadamente 45 puntos porcentuales. Esto, por supuesto, refleja la formalidad del trabajo específico. En contraste con las regresiones para el seguro, las variables dummy de los derechos laborales no muestran resultados sólidos para las regresiones con acceso a los servicios de salud. Solo el seguro de riesgo es significativo en las regresiones 5-8, lo que sugiere una correlación positiva con la probabilidad de tener acceso a la atención médica pública. Sin embargo, el efecto desaparece cuando se introduce el seguro de salud como una variable independiente, lo que significa que tener un seguro es uno de los determinantes más importantes de la probabilidad de acceder a la salud pública y captura la formalidad de las relaciones laborales explicada por la tenencia del seguro de riesgo en los modelos anteriores.

Para el seguro de salud, la categoría de ingreso solo es significativa en la regresión 4. Dicha variable se correlaciona positivamente con la probabilidad de tener seguro, lo que sugiere que cada aumento en la categoría de ingresos aumenta la probabilidad de seguro en 2,6 puntos porcentuales. En otras palabras, este aumento se produce cuando los ingresos aumentan en 50,000 colones (o aproximadamente US \$100) al mes, que es la diferencia entre las categorías 
construidas. Sin embargo, la regresión 5 sugiere que el mismo aumento de los ingresos reduce la probabilidad de acceder a los servicios de salud por un tamaño de probabilidad similar. Sin embargo, en general, el hecho de que la categoría de ingresos no tenga significación entre los modelos, pone en duda su importancia para el acceso al sistema de salud de Costa Rica entre los migrantes. De manera similar, la educación no tiene ninguna relación con la probabilidad de estar asegurado u obtener acceso a los servicios de salud.

La evidencia estadística para apoyar la afirmación de que los inmigrantes acceden a los servicios de salud a través de sus hijos, como lo sugiere el trabajo etnográfico, es muy débil en el mejor de los casos. La regresión 8 sugiere que hay un pequeño efecto positivo ejercido por la presencia de niños pequeños, controlando el seguro y el estado migratorio. Para obtener un seguro, sin embargo, no existe tal efecto. Finalmente, de acuerdo con el principio universal del seguro de salud y cobertura de servicios de salud de Costa Rica, no hay evidencia de que la probabilidad de estar asegurado o el acceso a los servicios sea diferente en las áreas urbanas y rurales.

\section{LA REGULARIZACIÓN: LA IMPORTANCIA DE TENERLA Y LA DIFICULTAD DE OBTENERLA}

En este artículo se ha analizado el acceso real de las personas inmigrantes nicaragüenses a los servicios de salud costarricenses, bajo la premisa, fundada en trabajo cualitativo, de que puede haber un acceso diferenciado entre nacionales e inmigrantes a los servicios de salud. El análisis se basó en datos de una encuesta entre población nicaragüense en Costa Rica, específicamente diseñada para capturar información sobre el acceso a seguro, y uso de los servicios sociales costarricenses.

El análisis demuestra que, efectivamente, existe un acceso diferenciado al seguro de salud y a los servicios de salud entre diferentes grupos de inmigrantes, y entre inmigrantes y nacionales. Esencial para la incorporación a los servicios de salud, es la obtención del seguro y el estado migratorio de la persona. Inmigrantes "ilegales", en este escenario, son los menos protegidos, mientras que los denizens son los más protegidos (incluso más que nacionales). Esto conlleva dos importantes mensajes para el debate público en Costa Rica. 
Primero, que la apuesta (por lo menos en el discurso formal) del Estado costarricense por direccionar la política pública a la regularización de inmigrantes, es la correcta si de acceso a salud se trata. Dado que la salud es posiblemente una de las dimensiones más importantes de la integración, estas son buenas noticias.

Segundo, las no tan buenas noticias son que en la realidad el Estado ha hecho más difícil tanto el proceso de la regularización ha creado mecanismos de exclusión del seguro social. El alto costo y el requisito de la afiliación al seguro social de la regularización a partir del 2010 con la entrada en vigor de la Ley de Migración 8764 del 2009, y, en el 2012, el requisito de la CCSS de tener un estado migratorio regular para obtener un seguro, son algunas de los mecanismos institucionales que han hecho más difícil el acceso al seguro y a los servicios de salud. Esto ayuda a explicar por qué existen desigualdades en las condiciones de acceso al y la cobertura del sistema de salud costarricense entre inmigrantes y nacionales. Así, el Estado, a pesar de priorizar discursivamente la regularización, en realidad parece hacer más difícil este mismo proceso (Sandoval, 2012; Fouratt, 2014).

En términos más generales, este artículo demuestra varias cosas importantes para los estudios de la política social y la migración, especialmente desde el Sur. Primero, que los Estados pueden relativamente fácilmente comprometerse con marcos normativos de derechos humanos de inclusión social, pero al mismo tiempo crear mecanismos de exclusión. Segundo, y por eso, es importante ampliar el estudio de la política social en general, y el acceso de migrantes a los servicios sociales en particular, al acceso real en vez de limitarlo al estudio de derechos formales y criterios de elegibilidad. Queda claro que, entre lo dicho formalmente, y lo accedido realmente, puede existir una brecha. Si bien desde hace tiempo se ha hecho énfasis en el estudio de la brecha de implementación (Faist, 1994; Morissens and Sainsbury, 2005; Morissens, 2008; Sainsbury, 2006), en América Latina, y el Sur en general, todavía hay un vacío en la investigación.

Tercero, en países en vías de desarrollo, la incorporación de inmigrantes en arreglos de bienestar es un punto sin mucha prioridad de la agenda pública, incluso en países como Costa Rica que tienen una larga historia de protección social universal. En muchos países con débiles regímenes de protección social o en países en los que sus fuertes regímenes se han visto debilitados por décadas de medidas 
de austeridad (como Costa Rica), la cobertura de servicios sociales a su población nacional ya representa un reto importante. En estos escenarios, la extensión de derechos y servicios sociales a migrantes no es una prioridad.

Finalmente, la política social puede fungir como un mecanismo que refuerza patrones de estratificación, y hace más complejo el reto de la integración de personas inmigrantes en la sociedad. Si la diferencia en el trato de nacionales e inmigrantes es la medida real de la recepción de inmigrantes, así como la posibilidad de hacer efectivo sus derechos, en muchos países en el mundo, Costa Rica incluida, queda todavía mucha tela por cortar.

\section{BIBLIOGRAFÍA}

Banco Mundial. (2017). Datos de libre acceso del Banco Mundial. Recuperado de: https://datos.bancomundial.org/indicador/SP.DYN.LE00. IN?end=2015\&locations=NL-NO-DE\&start=2015.

Banting, K. (2000). Looking in three directions: Migration and the European welfare state in comparative perspective. En M. Bommes \& A. Geddes (Eds.), Immigration and Welfare. Challenging the borders of the welfare state (pp. 13-33). London/New York: Routledge.

Bonilla-Carrión, R. (2007). Seguro social y usos de servicios de salud entre personas nicaragüenses en Costa Rica. En C. Sandoval (Ed.), El mito roto. Inmigración y emigración en Costa Rica (pp. 145-160). San José, Costa Rica: Editorial UCR.

CCSS (Caja Costarricense de Seguro Social). (2012a). Afiliación y prestación de servicios de salud a los asegurados extranjeros no habilitados legalmente para trabajar en el país. Comunicación interna, 10/04/2012. San José: CCSS.

CCSS (Caja Costarricense de Seguro Social). (2012b). Afiliación y prestación de servicios de salud a los asegurados extranjeros no habilitados legalmente para trabajar en el país. Comunicación interna, 22/06/2012. San José: CCSS

Castillo, J. (2003). Características de la Atención de los Extranjeros en los Servicios de Salud de la Caja Costarricense de Seguro Social 1997-2002. San José: CCSS, Presidencia Ejecutiva, Dirección Actuarial y de Planificación Económica.

Clark, M. (2013). The Final Frontiers of Healthcare Universalisation in Costa Rica and Panama. Bulletin of Latin American Research, 33(2), pp. 125-139.

Comisión Económica para América Latina y el Caribe (CEPAL). (2016). CEPALSTAT 2016. Recuperado de: http://estadisticas.cepal.org/cepalstat/ Portada.html. 
Comisión Económica para América Latina y el Caribe (CEPAL). (2017). "Estimaciones y proyecciones de población total, urbana y rural, y económicamente activa”. Recuperado de: https://www.cepal.org/es/temas/ proyecciones-demograficas/estimaciones-proyecciones-poblacion-totalurbana-rural-economicamente-activa.

Danzer, A., y Ulku, H. (2008). Determinants of Integration and its Impact on the Economic Success of Immigrants: A Case Study of the Turkish Community in Berlin. IZA DP No. 3762. Bonn: Institute for the Study of Labor.

Dirección General de Migración y Extranjería (DGME). (2011). Primer Informe Nacional de Migración e Integración. San José: DGME.

Dobles, I., Vargas, G., y Amador, K. (2013). Inmigración: Psicología, identidades y politicas públicas. La experiencia nicaragüense y colombiana en Costa Rica. San José, Costa Rica: Editorial UCR.

Faist, T. (1994). Immigration, integration and the ethnicization of politics. European Journal of Political Research, 25, pp. 439-459.

Filgueira, F. (1998). El nuevo modelo de prestaciones sociales en América Latina: residualismo, eficiencia y ciudadanía estratificada. En Roberts, B. (Ed.), Ciudadanía y Política Sociales. San José, Costa Rica: FLACSO/SSRC.

Fouratt, C., y Voorend, K. (2018). Sidestepping the State: Practices of Social Service Commodifcation among Nicaraguans in Costa Rica and Nicaragua. Journal of Latin American Studies, 50(2), pp. 441-468. DOI: 10.1017/ S0022216X17001195.

Fouratt, C. (2014). 'Those who come to do harm': The Framings of Immigration Problems in Costa Rican Immigration Law. International Migration Review, 48(1), pp. 144-180.

Goldade, K. (2009). 'Health Is Hard Here' or 'Health for All'? The Politics of Blame, Gender, and Health Care for Undocumented Nicaraguan Immigrants in Costa Rica. Medical Anthropology Quarterly, 23(4), pp. 483-503.

Goldade, K. (2011). Babies and Belonging: Reproduction, Citizenship, and Undocumented Nicaraguan Labor Migrant Women in Costa Rica. Medical Anthropology, 30(5), pp. 545-568.

González, H., y Varela, J. (2003). Entrevista telefónica. Heredia: Universidad Nacional, Instituto de Estudios Sociales en Población (IDESPO).

Instituto de Investigaciones Sociales (IIS); Centro de Investigación en Cultura y Desarrollo; Programa radiofónico Casa Abierta 870 UCR; Servicio Jesuita para Migrantes Costa Rica. (2012). La Ley de Migración en Costa Rica: A dos años de su entrada en vigencia. Promesas, realidades y desaf íos. Recuperado de: http://www.lanuevaprensacr.com/la-ley-de-migracion-en-costa-rica-a-dos-anosde-su-entrada-en-vigencia-promesas-realidades-y-des. Accesado 16-04-2013.

Instituto Nacional de Estadística y Censos (INEC). (1984). IX Censo Nacional de Población, Costa Rica 1984. San José: INEC.

Instituto Nacional de Estadística y Censos (INEC). (2000). IX Censo Nacional de Población. Costa Rica 2000. San José: INEC. 
Instituto Nacional de Estadística y Censos (INEC). (2004). Encuesta Nacional de Ingresos y Gastos, 2004. San José: INEC.

Instituto Nacional de Estadística y Censos (INEC). (2011). X Censo Nacional de Población, Costa Rica 2011. San José: INEC.

Kron, S. (2011). Gestión migratoria en Norte y Centroamérica: Manifestaciones y contestaciones. Anuario de Estudios Centroamericanos, 37, pp.53-85.

Martínez Franzoni, J., y Sánchez-Ancochea, D. (2013). Good jobs and social services: How Costa Rica achieved the elusive double incorporation. Hampshire: Palgrave Macmillan.

Martínez Franzoni, J. (2008). Domesticar la incertidumbre en América Latina: mercados laborales, política social y familias. San José, Costa Rica: Editorial UCR.

Money, J. (2010). Comparative Immigration Policy. En Denemark, R.A. (Ed.), The International Studies Encyclopedia (pp. 1-32). Chister, West Sussex, UK: Wiley-Blackwell.

Morales, A., y Castro, C. (2006). Migración, empleo y pobreza. San José: FLACSO-Costa Rica

Morissens, A., y Sainsbury, D. (2005). Migrants' Social Rights, Ethnicity and Welfare Regimes. Journal of Social Policy, 34(4), pp. 637-60.

Morissens, A. (2008). Migration, welfare states and the incorporation of migrants in different welfare regimes. UNRISD Flagship Report on Poverty. Geneva: UNRISD.

Naciones Unidas. (2012). Migrants by origin and destination: the role of South-South migration. Population Facts, Department of Economic and Social Affairs, Population Division. Recuperado de: http://www.un.org/ esa/population/publications/popfacts/popfacts_2012-3_South-outh_migration.pdf.

Noy, S., y Voorend, K. (2015). Social Rights and Migrant Realities: Migration Policy Reform and Migrants' Access to Health Care in Costa Rica, Argentina, and Chile. Journal of International Migration and Integration, 17(2), pp. 605-629.

Noy, S. (2012). World Bank Projects and Targeting in the Health Sector in Argentina, Costa Rica and Peru, 1980-2005. Artículo presentado en ISA 2012 Forum, Argentina: Buenos Aires, agosto 2.

Pribble, J. (2015). The Politics of Building Municipal Institutional Effectiveness in Chile. Latin American Politics and Society, 57(3), pp. 100-121.

Sainsbury, D. (2006). Immigrants' social rights in comparative perspective: welfare regimes, forms of immigration and immigration policy regimes. Journal of European Social Policy, 16(3), pp. 229-244.

Sandoval García, C. (2004). Contested Discourses on National Identity: Representing Nicaraguan Immigration to Costa Rica. Bulletin of Latin American Research, 23, pp. 434-445.

Sandoval García, C. (2012). Contestar la hostilidad antiinmigrante en Costa Rica. Un proyecto de Ciencias Sociales públicas en curso. En González, 
M. L. (Ed.), Entreverse. Teoría y metodología práctica de las fuentes orales (pp. 217-240). Bilbao: Universidad del País Vasco / Euskal Herriko Unibertsitatea, Argitalpen Zerbitzua.

Spesny-Dos Santos, S. (2015). Undeserving mothers? Shifting rationalities in the maternal healthcare of undocumented Nicaraguan migrants in Costa Rica. Anthropology \& Medicine, 22(2), pp.191-201.

Voorend, K., y Robles Rivera, F. (2011). Migrando en la crisis: La fuerza de trabajo inmigrante en la economía costarricense. San José: OIM, MTSS, Gobierno de Canadá.

Voorend, K. (2019). ¿Un imán de bienestar en el Sur? Migración y política social en Costa Rica. San José: Editorial UCR. 